\title{
Clinical and experimental evidence suggest a link between KIF7 and C5orf42-related ciliopathies through Sonic Hedgehog signaling
}

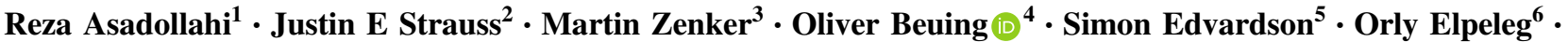 \\ Tim M Strom ${ }^{7} \cdot$ Pascal Joset $^{1} \cdot$ Dunja Niedrist $^{1} \cdot$ Christine Otte $^{1} \cdot$ Beatrice Oneda $^{1} \cdot$ Paranchai Boonsawat $^{1}$. \\ Silvia Azzarello-Burri ${ }^{1} \cdot$ Deborah Bartholdi $^{1} \cdot$ Michael Papik $^{1} \cdot$ Markus Zweier $^{1} \cdot$ Cordula Haas $^{8} \cdot$ Arif B Ekici $^{9} \cdot$ \\ Alessandra Baumer ${ }^{1} \cdot$ Eugen Boltshauser $^{10} \cdot$ Katharina Steindl $^{1} \cdot$ Michael Nothnagel $^{11} \cdot$ Albert Schinzel $^{1}$. \\ Esther T Stoeckli ${ }^{2,12} \cdot$ Anita Rauch $\mathbb{D}^{1,12,13}$
}

Received: 26 January 2017 / Revised: 11 September 2017 / Accepted: 19 September 2017 / Published online: 10 January 2018

(C) The Author(s) 2018. This article is published with open access

\begin{abstract}
Acrocallosal syndrome (ACLS) is an autosomal recessive neurodevelopmental disorder caused by KIF7 defects and belongs to the heterogeneous group of ciliopathies related to Joubert syndrome (JBTS). While ACLS is characterized by macrocephaly, prominent forehead, depressed nasal bridge, and hypertelorism, facial dysmorphism has not been emphasized in JBTS cohorts with molecular diagnosis. To evaluate the specificity and etiology of ACLS craniofacial features, we performed whole exome or targeted Sanger sequencing in patients with the aforementioned overlapping craniofacial appearance but variable additional ciliopathy features followed by functional studies. We found (likely) pathogenic variants of KIF7 in 5 out of 9 families, including the original ACLS patients, and delineated 1000 to 4000 -year-old Swiss founder alleles. Three of the remaining families had (likely) pathogenic variants in the JBTS gene C5orf42, and one patient had a novel de novo frameshift variant in $S H H$ known to cause autosomal dominant holoprosencephaly. In accordance with the patients' craniofacial anomalies, we showed facial midline widening after silencing of C5orf42 in chicken embryos. We further supported the link between KIF7, SHH, and C5orf42 by demonstrating abnormal primary cilia and diminished response to a SHH agonist in fibroblasts of C5orf42-mutated patients, as well as axonal pathfinding errors in C5orf42silenced chicken embryos similar to those observed after perturbation of Shh signaling. Our findings, therefore, suggest that beside the neurodevelopmental features, macrocephaly and facial widening are likely more general signs of disturbed SHH signaling. Nevertheless, long-term follow-up revealed that C5orf42-mutated patients showed catch-up development and fainting of facial features contrary to KIF7-mutated patients.
\end{abstract}

Reza Asadollahi, Justin E Strauss, Albert Schinzel, Esther T Stoeckli and Anita Rauch contributed equally to this work

Electronic supplementary material The online version of this article (https://doi.org/10.1038/s41431-017-0019-9) contains supplementary material, which is available to authorized users.

Publisher's note Springer Nature remains neutral with regard to jurisdictional claims in published maps and institutional affiliations.

Anita Rauch

anita.rauch@medgen.uzh.ch

Extended author information available on the last page of the article

\section{Introduction}

Acrocallosal syndrome (ACLS; [MIM \#200990]) is a neurodevelopmental ciliopathy which was first described in two unrelated Swiss patients with intellectual disability (ID), agenesis of the corpus callosum, craniofacial dysmorphism, and polydactyly together with additional anomalies [1, 2]. In 2011, biallelic mutations of KIF7 [MIM *611254], a key component of the Sonic Hedgehog (SHH) signaling pathway, have been found as the genetic cause of ACLS and fetal hydrolethalus syndrome [3]. Following the discovery of the gene and description of further patients showing overlapping features with Joubert syndrome (JBTS; [MIM \#213300]), ACLS has been listed under the designation of the clinically and genetically heterogeneous Joubert 
syndrome-related disorders (JSRD) [4, 5]. Distinct craniofacial features of macrocephaly, prominent forehead, and depressed/wide nasal bridge have been considered characteristic of ACLS [1-3, 6, 7], but were also emphasized in a fraction of molecularly undiagnosed JSRD cohorts [8-10]. Therefore, to study the specificity and etiology of the ACLS craniofacial features, we evaluated 13 cases from 9 families with the craniofacial appearance fitting into the ACLS spectrum as well as variable additional ciliopathy features by whole-exome sequencing (WES) or targeted Sanger sequencing of KIF7. Remarkably, 8 of the 9 families showed either KIF7 or C5orf42 [MIM *614571] recessive (likely) pathogenic variants, and in 1 patient we found a de novo frameshift variant of SHH [MIM *600725] indicating a shared role of these genes in craniofacial and neural development. To date, C5orf 42 has been reported as the most common mutated gene in JBTS [11, 12] and the related oral-facial-digital syndrome type VI (OFDVI) [13] often presenting with polydactyly and ocular motor apraxia beside the constant molar tooth sign (MTS)/vermis hypoplasia and ID/developmental delay (DD). Midline facial defects such as notched upper lip, cleft lip/ palate, and tongue hamartomas are additional features in OFDVI [13].

Since in contrast to KIF7 and SHH, the function of C5orf42 was unknown, we evaluated the primary cilia and their response to SHH agonist stimulation in fibroblasts of C5orf42-mutated patients, and assessed its role in development by in ovo RNAi-mediated gene silencing in chicken embryos. Our data provide the first evidence for the importance of C5orf42 in craniofacial development as well as axon guidance and also suggest its functional link to KIF7 and SHH through SHH signaling. Taken together, the craniofacial features previously considered as characteristic of ACLS appear to be a more general sign of disturbed SHH signaling.

\section{Subjects and Methods}

\section{Patients}

We collected 13 cases from nine families including the original ACLS cases reported by Schinzel et al. [2, 14], presenting with relative/absolute macrocephaly, broad/prominent forehead, depressed/wide nasal bridge and hypertelorism as well as variable additional ciliopathy or ACLS features including polydactyly, MTS/cerebellar vermis hypoplasia, abnormal corpus callosum, oculomotor apraxia, and notched upper lip (Table 1). Genetic testing was either performed on a diagnostic basis with subsequent consent for publication or as part of a research study approved by the ethics commission of the Canton of Zurich.

\section{Genetic studies}

Genetic studies were performed on DNA extracted from peripheral blood except for the deceased father of patient 13 , in whom the DNA was extracted from shaving material using the standard Chelex method [15]. In the latter, the assumed probe relationship was confirmed as described in the Supplementary Materials and Methods. Targeted Sanger sequencing of KIF7 (NM_198525.2, NG_030338.1) in patients $2,3,9,10$, and 13 , and the mother of patient 1 was performed after PCR amplification of all exons and flanking intronic nucleotides using an AB3730 capillary sequencer (Applied Biosystems, Foster City, CA). Candidate nucleotide variants from WES in C5orf42 (NM_023073.3, NG_032772.1) and SHH (NM_000193.2, NG_007504.1) were also confirmed by Sanger sequencing. Variant nomenclature, and variant classification are based on the HGVS recommendations (http://varnomen.hgvs.org/), and ACMG guidelines [16], respectively. Chromosomal microarray analysis in patients $2-5,9-11,13$ and the mother of patient 1, was performed using Affymetrix Cytoscan HD arrays as described previously [17]. WES in patients 4-12 was performed as described elsewhere with minor modifications [18], or using the Agilent SureSelect XT Clinical Research Exome Kit on a Illumina 2500 System (Illumina, San Diego, CA) in patient 13 and his mother. The WES data of individual cases were analyzed using the NextGENe Software (SoftGenetics, State College, PA) for non-silent exonic and splice site variants considering both dominant and recessive modes of inheritance. All variants were submitted to ClinVar database (https://www.ncbi.nlm. nih.gov/clinvar/) with ClinVar accessions SCV000579218, SCV000579459-68, and SCV000579219 for KIF7, $\mathrm{C} 5$ orf42, and $\mathrm{SHH}$, respectively. Variants of C5orf42 detected in patients 9-12 were also submitted to LOVD database (www.LOVD.nl/C5orf42) with IDs of 0010365053 , respectively.

We estimated the age of Swiss founder alleles using the ESTIAGE software [19] (Supplementary Materials and Methods) on haplotypes of patients 2 and 3, as well as two previously published [7] Swiss patients.

\section{Functional studies of $\mathrm{C} 5$ orf 42 in patient fibroblasts}

To assess the effect of the C5orf 42 variants on primary cilia and SHH signaling, standard skin-punch biopsies from patients 11 and 12 with (likely) pathogenic C5orf42 variants, their carrier parents and an unrelated control were obtained and cultured according to standard protocols. We quantified the number and length of primary cilia in the fibroblasts, and assessed their responsiveness to serum starvation and stimulation with a SHH agonist, SAG, as described in the Supplementary Materials and Methods. 


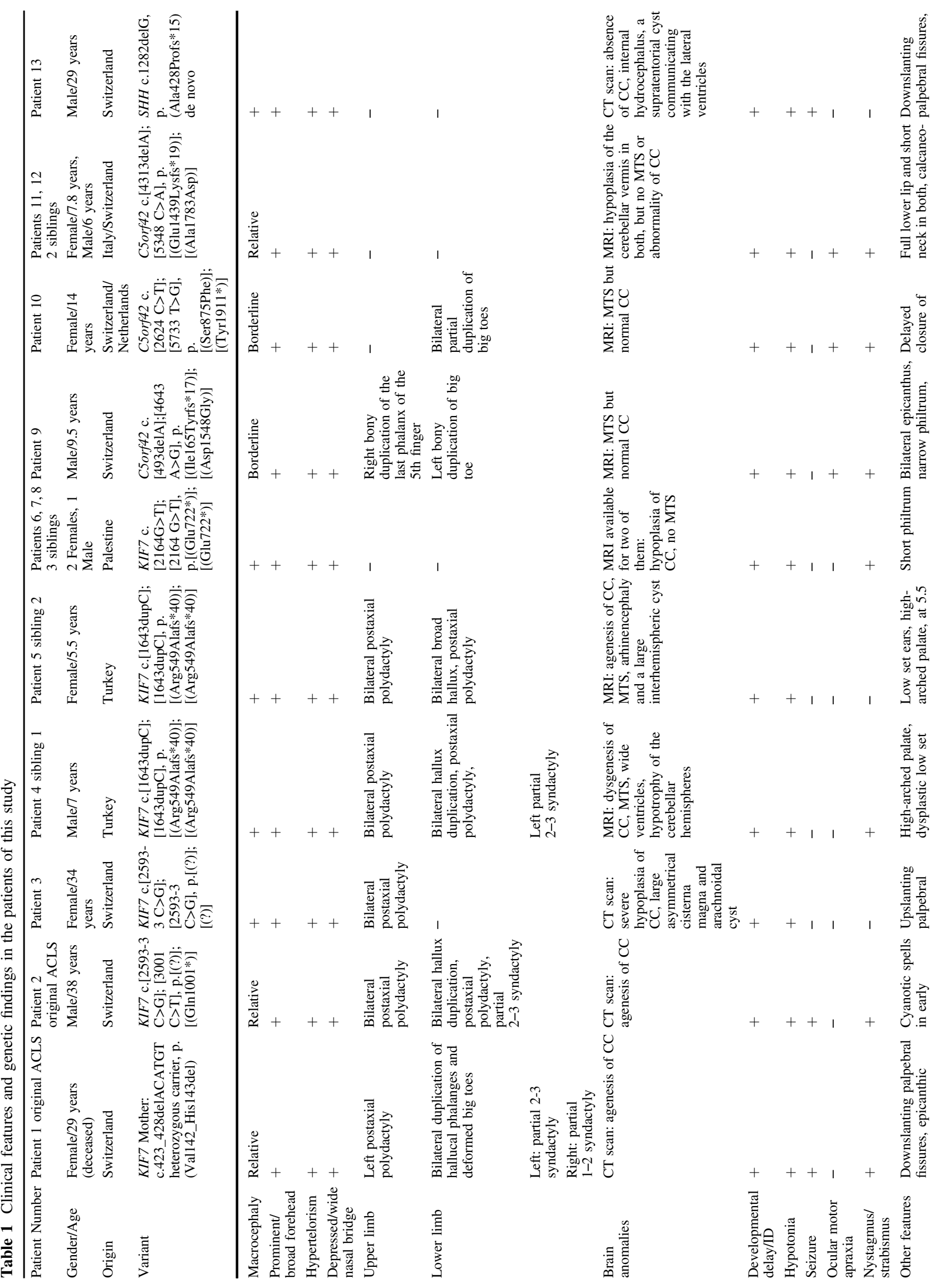








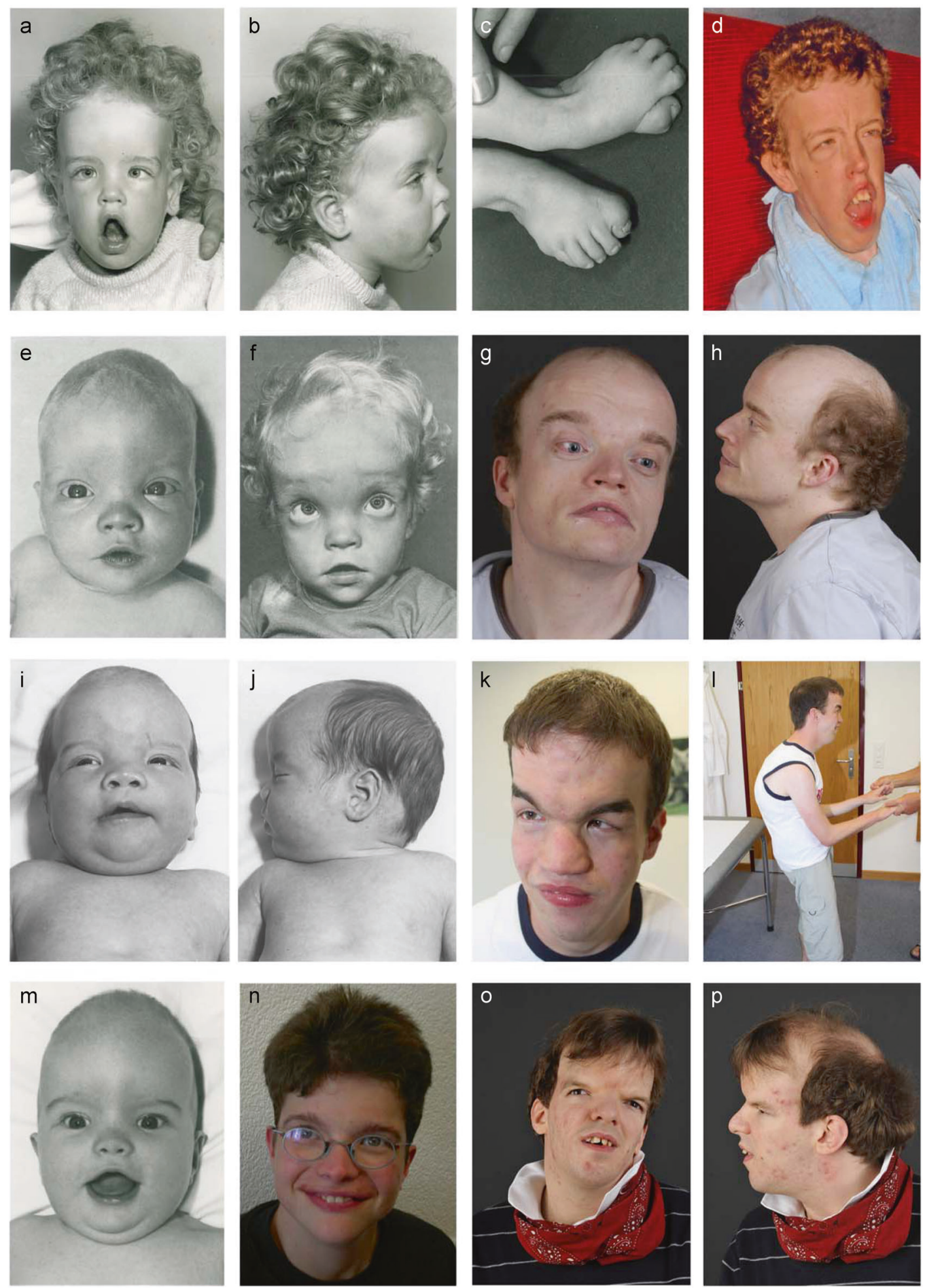

Fig. 1 Craniofacial features of patients with biallelic KIF7 or de novo $S H H$ variants. a-d Deceased female patient 1 at 2 years and 2 months a-c and in adulthood $\mathbf{d}$ whose mother was shown to be a heterozygous carrier of a KIF7 variant; e-h male patient 2 with pathogenic KIF7 biallelic Swiss founder variants at 3 months (e), 3 years (f) and 33 years $(\mathbf{g}, \mathbf{h})$ of age; $\mathbf{i}-\mathbf{l}$ the previously reported male patient (case 4 of Putoux et al. 2012) with the same biallelic variants as patient 2 at the age of 3 months $(\mathbf{i}, \mathbf{j})$ and 26 years $(\mathbf{k}, \mathbf{l}) ; \mathbf{m}, \mathbf{n}$ female patient 3

with KIF7 homozygous Swiss splice site pathogenic variants at 5 months $(\mathbf{m})$ and 21 years of age (n); $\mathbf{o}-\mathbf{p}$ male patient 13 with a de novo $\mathrm{SHH}$ variant at 29 years. Note the shared facial features of broad and high forehead, hypertelorism, flat nasal root, thin upper and everted lower lips, and retracted but relatively large chin. Patient 3 (m, n) shows the mildest dysmorphism corresponding with the milder intellectual disability, likely due to the leakiness of the splice site variant 


\section{Functional studies of C5orf42 in chicken embryos}

Gene silencing by in ovo RNAi

Loss-of-function phenotypes of $\mathrm{C} 5 \mathrm{orf} 42$ were induced by in ovo RNAi in chicken embryos as described before $[20,21]$ through the injection of double-stranded RNA followed by electroporation at Hamburger Hamilton (HH) [22] stages HH10-12 (embryonic day (E) 1.5-2) and HH17-19 (E2.5-3) to target cranial neural crest cells (NCCs), and dorsal commissural neurons (dI1) of the spinal cord, respectively. Untreated embryos, and embryos electroporated with a green fluorescent protein (GFP)-encoding plasmid were used as controls. Double-stranded RNA was
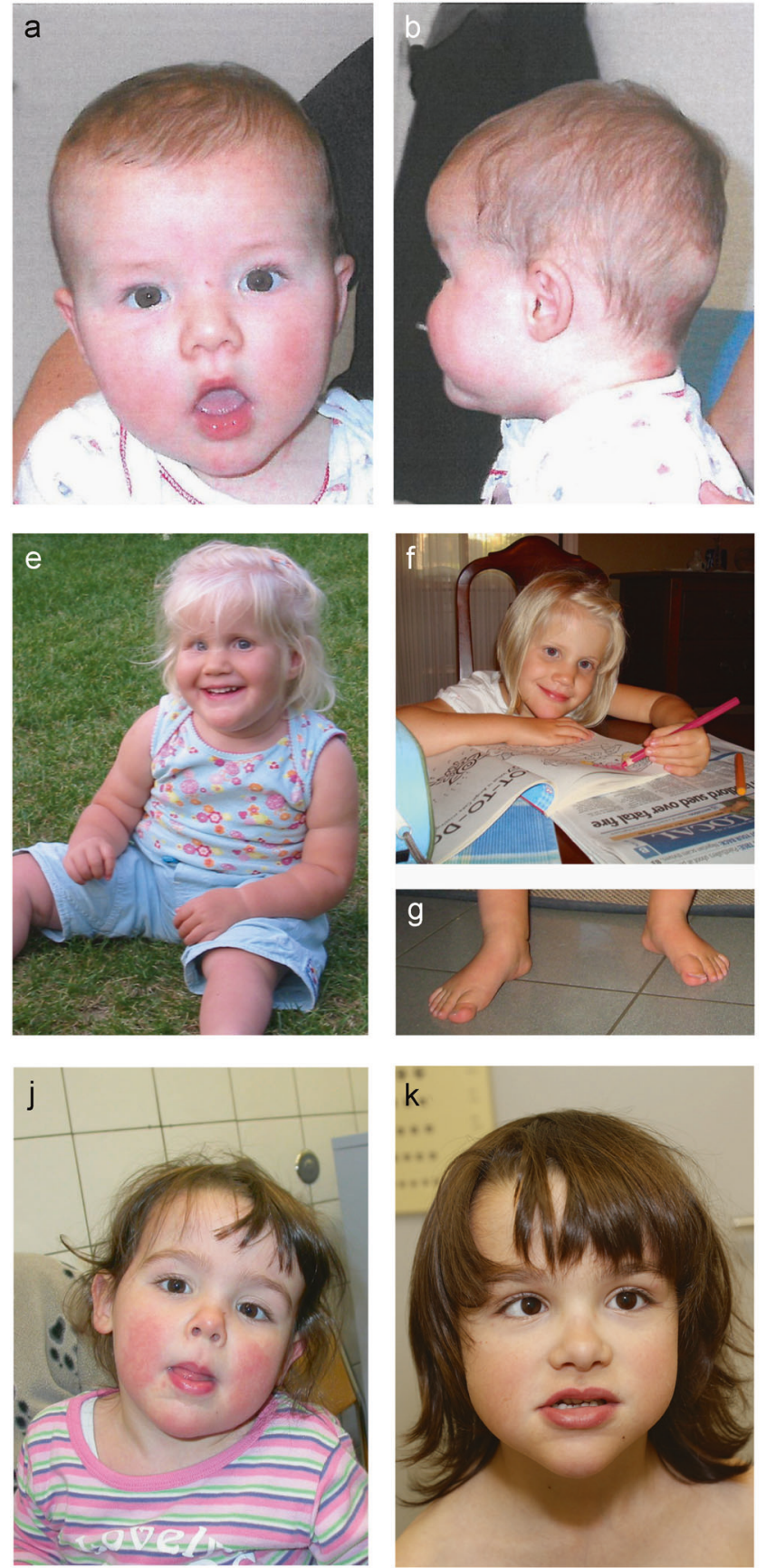

Fig. 2 Clinical features of patients with C5orf42 biallelic variants. a-d male patient 9 at 1 year 3 months (a-c) and 9.5 years (d); $\mathbf{e}-\mathbf{i}$ female patient 10 at 2 years 8 months (e), about 6.5 years (f) and about 14 years $(\mathbf{h}, \mathbf{i})$; j-k female patient 11 at about $2.5(\mathbf{j})$ and $7.5(\mathbf{k})$ years of
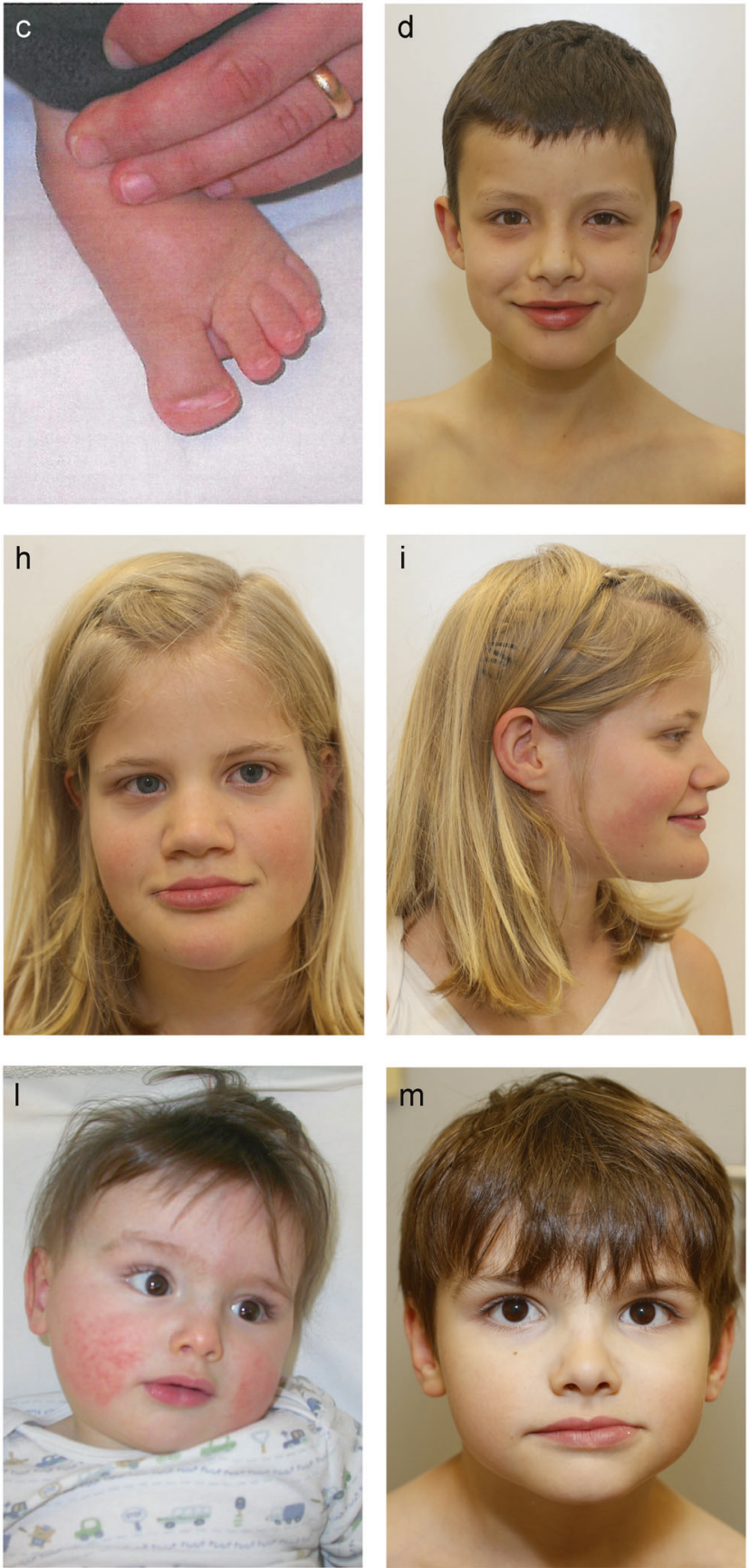

age; $\mathbf{l}-\mathbf{m}$ patient 12 (brother of patient 11) at 8 months (l) and 6 years (m) of age. Note the hallux duplication in patients 9 (c) and 10 (g) and ACLS-like craniofacial features in all patients being more evident in early childhood but becoming milder with time 
Fig. 3 Evaluation of the primary cilia and response to the $\mathrm{SHH}$ agonist SAG in fibroblasts of patients 11 and 12 with compound heterozygous (likely) pathogenic variants in C5orf 42 compared to the controls. a, b Representative fibroblast images from a control and patient 12 immunostained for acetylated- $\alpha$ tubulin (green). c, d Fibroblast cultures of the patients showed significantly fewer and shorter primary cilia compared to those of the controls. Results are expressed as mean values $\pm \mathrm{SD}$ $(* P<0.05, * * P<0.001$, Mann-Whitney). e, f qPCR analysis of GLII and PTCH1 expression in control and patient fibroblasts following $48 \mathrm{~h}$ serum starvation and subsequent $48 \mathrm{~h}$ treatment with SAG. After treatment, patient fibroblasts showed significantly lower level increase in the expression of GLII and PTCH1 transcripts compared to the controls. Results are expressed as mean values \pm SEM $(* P<0.05, * * P$ $<0.001, t$ test) a

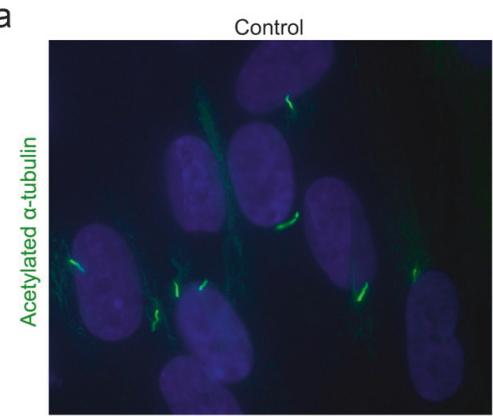

C

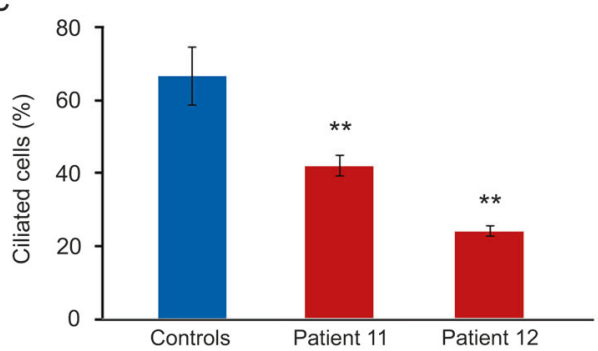

e

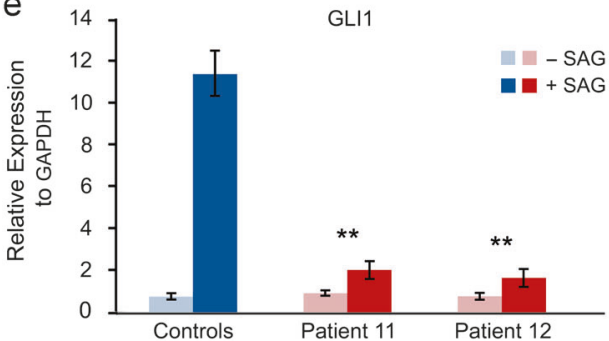

b

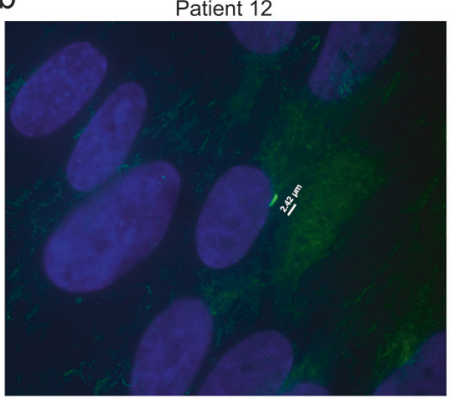

d

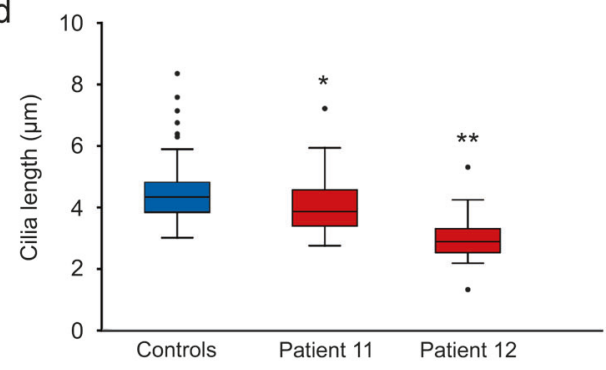

f

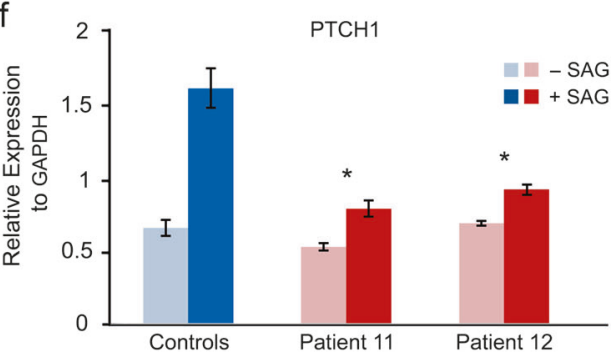

derived from two non-overlapping fragments of the C5orf42 open reading frame (XM_015277526).

\section{Analysis of dorsal commissural axon pathfinding}

For analysis of commissural axon trajectories, embryos were killed at HH25-26 (E5). The spinal cord was removed from the embryo, opened at the roof plate (open-book preparation) and fixed [23]. The trajectories of dorsal commissural axons (dI1) at the lumbar level of the spinal cord were visualized using the lipophilic dye Fast-DiI applied to the cell bodies [21]. Pathfinding behavior of dI1 commissural axons was categorized as "normal" when axons crossed the midline and turned rostrally along the contralateral floor-plate border, as "stalling" if at least $50 \%$ of the axons failed to cross the midline, or as "no turn" if at least $50 \%$ of the axons reaching the contralateral floor-plate border failed to turn rostrally. The data from experimental, control-injected, and untreated control embryos were compared using analysis of variance followed by Tukey's posthoc test accessed on the VassarStats Website for Statistical Computation (http://vassarstats.net/).
Analysis of neural circuit formation in the peripheral nervous system

For analysis of dorsal root ganglia (DRG) and dorsal roots, sciatic nerve, and crural nerve, embryos were killed at HH25-26 (E5) and stained as whole mounts using an antineurofilament antibody (RMO270; 1:1500; Invitrogen) [22].

\section{Analysis of craniofacial features}

To analyze the role of C5orf42 in craniofacial development, we silenced C5orf42 in cranial NCCs at HH10-12. Heads of embryos killed at $\mathrm{HH} 29$ were incubated with Alcian Blue solution $(0.02 \%$ Alcian Blue in $80 \%$ ethanol, $20 \%$ acetic acid) for $24 \mathrm{~h}$ at $4^{\circ} \mathrm{C}$ on an orbital shaker. The heads were then transferred to pure ethanol for 5 days at $4{ }^{\circ} \mathrm{C}$ to remove excessive dye. After incubation overnight in $0.5 \% \mathrm{KOH}$ and several days in $1.8 \% \mathrm{KOH}$, heads were imaged with an Olympus SZX12 stereo microscope. 
Fig. 4 Silencing C5orf42 in the developing neural tube of chicken embryos resulted in pathfinding errors of commissural axons at the midline. The pathfinding behavior of dI1 commissural axons at the floor plate was analyzed in open-book preparations of the spinal cord (a) see Methods section for details). Axonal trajectories (red) are visualized by the injection of DiI into the area of commissural neuron cell bodies. In untreated control embryos, commissural axons grew ventrally, entered the floor plate to cross the midline and turned rostrally at the floor-plate exit site (arrows, b). No difference in commissural axon pathfinding was observed in control-treated embryos (c). In contrast, after silencing C5orf42 in the neural tube, dI1 commissural axons failed to cross the midline, stalled in the floor plate (arrow heads) and also failed to turn rostrally along the contralateral floor-plate border (open arrow, d). The floor plate is indicated by dashed lines. Bar: $50 \mu \mathrm{m}$. Quantification of phenotypes as average percentage of DiI injection sites per embryo with the given phenotypes (e, shown with SEM; $* P<0.05)$. Green bars for untreated control embryos, blue bars for controltreated embryos-expressing GFP, red bars for embryos after RNAi-mediated silencing of C5orf42 (LOF, loss-offunction). In addition, silencing C5orf42 in cranial neural crest cells of developing chicken embryos resulted in facial dysmorphism. In comparison with the control-treated embryos (f), embryos lacking C5orf42 in cranial neural crest cells developed aberrant facial features $(\mathbf{g})$. The nasal structure (arrowhead) was much wider than in age-matched control heads stained with Alcian Blue. Similarly, the jaw was broader in experimental compared to control embryos (indicated by black line) and eye distance was increased a
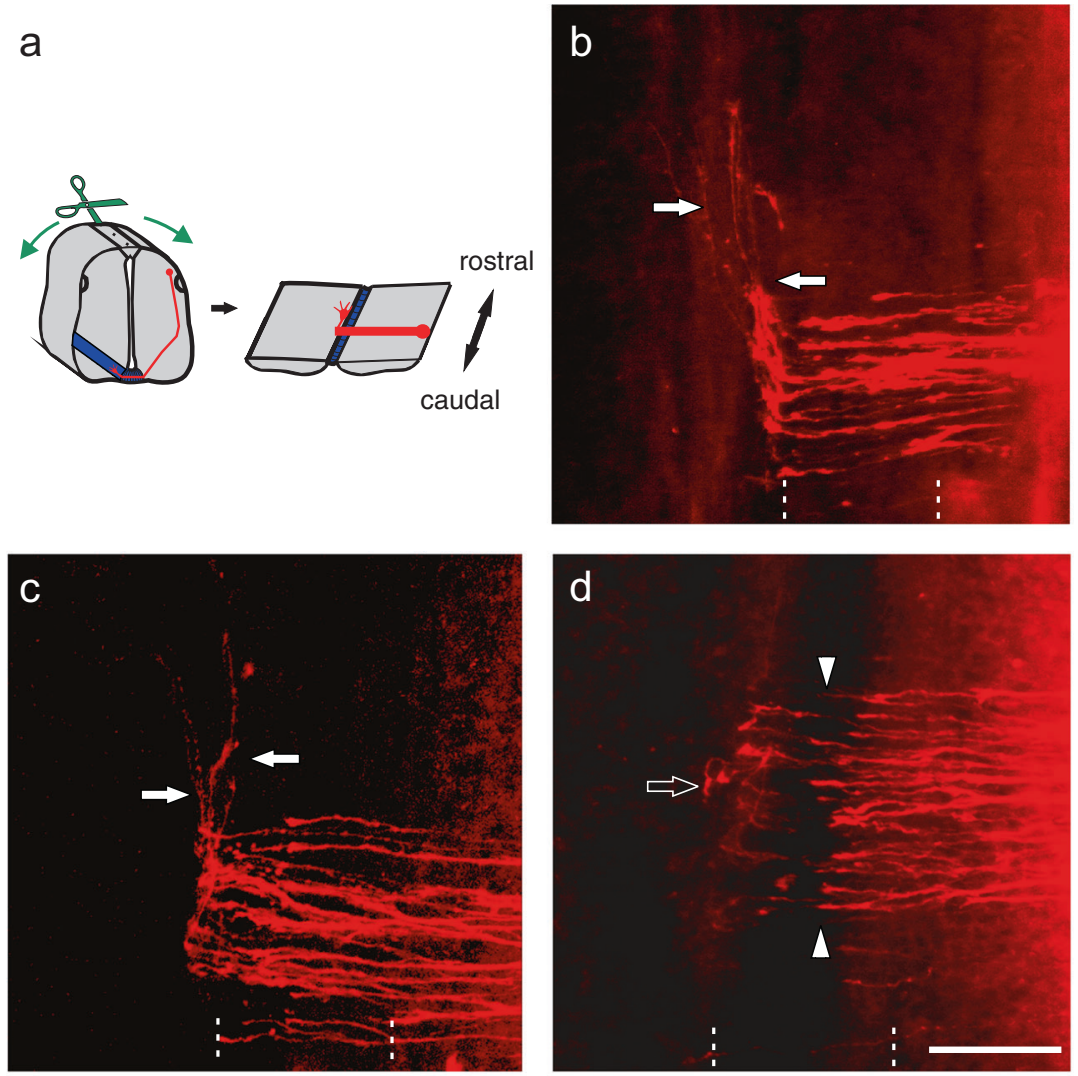

e

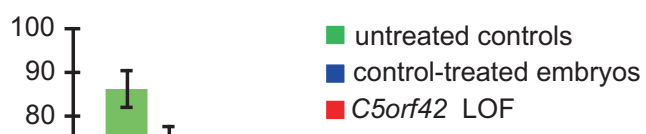

f

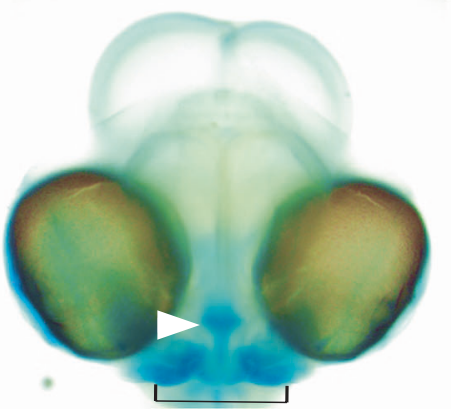

g

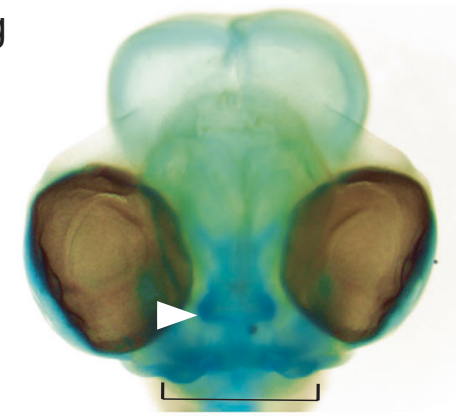




\section{Results}

\section{Genetic studies in the patients}

Targeted Sanger sequencing or WES revealed homozygous or compound heterozygous KIF7 pathogenic variants in 7 patients (patients 2-8). In the deceased patient 1 , only the DNA from the mother was available, who was confirmed by Sanger sequencing to be the carrier of a heterozygous KIF7 variant. By WES, we found compound heterozygous (likely) pathogenic variants affecting C5orf42 in 4 patients (patients 9-12), and a de novo frameshift variant affecting SHH in 1 patient (patient 13). Summary of all the variants and their classification are available in Table 1 and Supplementary Table S1.

\section{Description of patients and variants}

\section{Patients 1 to 8 with ACLS harboring KIF7 variants}

Patients 1-3 were the two original patients $[1,2]$ and a later described case [14] with ACLS reported by Schinzel et al. in 1980 and 1988, respectively. Their follow-up clinical information, morphological anomalies and sequencing results are summarized in Table 1, Supplementary Results and Fig. 1. We identified a heterozygous deletion of two amino acids in the kinesin motor domain of KIF7 in the mother of patient 1 , as the only available family member. Patients 2 and 3 harbored the same two splice site and stop pathogenic variants reported previously [7] in two other Swiss patients (one of them depicted in Fig. 1i-l). Of note, all carriers of the splice site variant (c.2593-3 C>G) originated from the Cantons of Appenzell in Northeastern Switzerland, and those carrying the stop variant (c.3001 C > T; p. $\left.\left(\mathrm{Gln} 1001^{*}\right)\right)$ originated from the Canton of Lucerne in central Switzerland (Supplementary Fig. S1). We estimated the most recent common ancestor (MRCA) of the c.2593-3 C $>\mathrm{G}$ variant, based on haplotype sharing analysis of three variant-carrying haplotypes, to have lived 159 generations ago (95\% CI: 65-415), corresponding to the point estimates of the mutational age of 2385,3180 , or 3975 years when assuming a generation time of 15,20 , or 25 years, respectively. The c.3001 C $>\mathrm{T}$ (p.(Gln1001*)) variant, based on the analysis of five haplotypes, is estimated to be much younger, with the MRCA to have lived 78 generations (95\% CI: 40-154) ago, translating into 1170-1950 years before present with generation times between 15 and 25 years, respectively.

Patients $4-5$ and 6-8 were offsprings of consanguineous parents harboring homozygous frameshift or stop variants in KIF7 (Table 1), respectively, the former located within a shared long stretch of homozygosity. Their clinical information is summarized in Table 1, Supplementary Results and Fig. S2.
Patients 9 to 12 with JBTS harboring C5orf42 variants

Both of these affected sib pairs (patients 9-10 and 11-12) were offspring of non-consanguineous parents with compound heterozygous variants in C5orf42. Their clinical information, morphological anomalies and sequencing results are summarized in Table 1, Supplementary Results and Fig. 2. Notably, we found that C5orf42 biallelic mutant fibroblasts from patients 11 and 12 formed significantly fewer and shorter primary cilia compared to the control fibroblasts $(P<0.05$, Mann-Whitney) (Fig. 3a-d). After treatment with SAG, patient fibroblasts showed significantly lower level increase in the expression of GLII and PTCH1 transcripts compared to the controls $(P<0.05$, $t$ test) (Fig. 3e-f)

\section{Patients 13 harboring a SHH variant}

Patient 13 was offspring of non-consanguineous parents and was negative for KIF7 and C5orf42 variants but harbored a previously unreported de novo frameshift variant in the last exon (exon 3) of SHH. His clinical information, morphological anomalies, and sequencing result are summarized in Table 1, Supplementary Results and Fig. 1o, p. This variant causes a frameshift with introduction of a premature stop codon, but affects the last 35 amino acids at C-terminal and therefore likely does not lead to nonsense-mediated mRNA decay. Nevertheless, the C-terminal portion of SHH is essential for catalyzing the autocleavage of the protein to the active $\mathrm{N}$-terminal fragment, as well as additional posttranslational modifications [24], and therefore we assume that the modified C-terminus interferes with these processes leading to abnormal levels of active SHH. However, further patients with similar phenotype and $\mathrm{SHH}$ variants are needed to clarify the significance of this variant.

\section{Functional analysis of C5orf42 in chicken embryos}

To decipher the role of C5orf42 in neural and craniofacial development, we evaluated the phenotypic changes following its silencing in chicken embryos. We found crucial roles of C5orf42 in axonal pathfinding in the central nervous system (CNS) (Fig. 4), and in the peripheral nervous system (PNS) (Supplementary Fig. S3), as well as craniofacial development in accordance with the phenotypes seen in the patients (Fig. $4 \mathrm{~h}-\mathrm{j}$ ).

\section{Silencing of C5orf42 interferes with the pathfinding of commissural axons}

In untreated and control-treated embryos commissural dI1 axons had crossed the midline and turned rostrally along the contralateral floor-plate border at $\mathrm{HH} 25-26$ (Fig. 4a-c). 
Table 2 Frequency of major clinical features in patients with recessive $K I F 7$ or $C 5$ orf 42 variants from the literature and this study

\begin{tabular}{lll}
\hline Clinical feature & $\begin{array}{l}\text { KIF7-positive } \\
\text { ACLS/ JBTS }\end{array}$ & $\begin{array}{l}\text { C5orf42-positive } \\
\text { JBTS/ OFDVI }\end{array}$ \\
\hline (Relative) Macrocephaly & $28 / 34(82 \%)$ & $5 / 5(100 \%)$ \\
Prominent/broad forehead & $34 / 38(89 \%)$ & $9 / 9(100 \%)$ \\
Hypertelorism & $34 / 37(92 \%)$ & $7 / 8(88 \%)$ \\
$\begin{array}{l}\text { Depressed/wide nasal } \\
\text { bridge }\end{array}$ & $33 / 34(97 \%)$ & $6 / 6(100 \%)$ \\
Polydactyly & $26 / 41(63 \%)$ & $37 / 71(52 \%)$ \\
Abnormal corpus & $38 / 40(95 \%)$ & $6 / 13(46 \%)$ \\
callosum & & \\
MTS/VH & $18 / 32(56 \%)$ & $77 / 77(100 \%)$ \\
Developmental delay/ID & $40 / 41(98 \%)$ & $36 / 36(100 \%)$ \\
Seizure & $7 / 18(39 \%)$ & $2 / 19(11 \%)$ \\
Ocular motor apraxia & $0 / 9(0 \%)$ & $27 / 38(71 \%)$ \\
Nystagmus/strabismus & $9 / 18(50 \%)$ & $13 / 21(62 \%)$ \\
JBTS breathing & $1 / 10(10 \%)$ & $14 / 28(50 \%)$ \\
abnormalities & & \\
\hline
\end{tabular}

$A C L S$ acrocallosal syndrome, JBTS Joubert syndrome, OFDVI oralfacial-digital syndrome type VI

MTS molar tooth sign, $V H$ vermis hypoplasia, $I D$ intellectual disability

After silencing C5orf42 the initial growth of dI1 axons towards the floor plate was not different from controls and axons reached the ventral midline of the neural tube at the appropriate time. However, in experimental embryos axons either failed to cross the midline or to turn rostrally along the contralateral border of the floor plate (Fig. 4d, e). On average, we found axonal stalling at $22.7 \pm 4.9 \%$ of the injection sites in C5orf42-deficient embryos, compared to $2.7 \pm 1.4$ and $1.8 \pm 1.0 \%$ in untreated and GFP-expressing controls, respectively. Furthermore, the failure of dI1 axons to turn rostrally along the contralateral floor-plate border was observed at an average of $42.9 \pm 5.9 \%$ of the injection sites after C5orf42-silencing compared to $9.7 \pm 4.3 \%$ and $26.6 \pm 5.3 \%$ in the untreated and GFP-expressing controls, respectively. Overall, after silencing of $C 5$ orf 42 , on average only $44.6 \pm 5.7 \%$ of the analyzed injection sites per embryo showed normal axon guidance which was significantly lower than the $86.2 \pm 4.0 \%$ in untreated $(P<0.05)$ and 72.3 $\pm 5.2 \%$ in GFP-expressing controls $(P<0.05)$ (Fig. $4 \mathrm{e})$.

\section{Silencing C5orf42 affects neural circuit formation in PNS}

After silencing C5orf42, the size and arrangement of DRG, the number of dorsal roots, and the branching of sciatic nerves were perturbed in embryos analyzed at $\mathrm{HH} 25-26$ (Supplementary Fig. S3). After loss of C5orf42 function, DRG were of variable shape and size, and they were arranged asymmetrically in $>90 \%$ of the embryos (Supplementary Fig. S3B, G). In addition, adjacent DRG were connected by fibers or had joint roots, aberrant phenotypes that were not observed in control embryos (Supplementary Fig. S3C).

Neural circuits in the limbs were compromised in the absence of C5orf42. In control embryos, the dorsal branch of the sciatic nerve segregated into an anterior and a posterior branch of about the same thickness (Supplementary Fig. S3D). In contrast, experimental embryos showed aberrant sciatic nerve branching (Supplementary Fig. S3E).

Silencing of C5orf42 in cranial NCCs leads to abnormal facial features in chicken embryos

After silencing C5orf42 in cranial NCCs, embryos developed aberrant facial features compared to controls (Fig. 4f-g). The distance between the eyes was increased, their nasal structure and their jaw were much wider compared to the age-matched controls.

\section{Discussion}

Genetic analysis in our cohort of patients with craniofacial appearance fitting into the ACLS clinical spectrum and variable additional ciliopathy features revealed (likely) pathogenic variants not only in KIF7, but also secondly in C5orf42, the most commonly mutated gene in JBTS [11, 12] and the related OFDVI [13]. In addition, in one patient we found a heterozygous de novo frameshift variant in $\mathrm{SHH}$, the most common cause of non-chromosomal holoprosencephaly (HPE) [25, 26]. All our patients shared ID, relative or absolute macrocephaly, prominent/broad forehead, depressed/wide nasal bridge, hypertelorism, and brain anomalies of either abnormal corpus callosum or MTS or vermis hypoplasia. However, long-term follow-up revealed that patients with pathogenic KIF7 variants had more severe and persistent facial dysmorphism and in general more severe ID, while in patients with (likely) pathogenic C5orf42 variants, facial dysmorphism became less prominent over time and they could catch up and reach a level of mild to moderate ID despite initial severe DD. Importantly, patient 3 with homozygosity of the KIF7 splice site pathogenic variant at position -3 could also catch up with only mild cognitive deficits in adulthood, which may indicate some leakiness of this variant. Notably, C5orf42 (likely) pathogenic variants are nearly invariably associated to the MTS/vermis hypoplasia and less often to corpus callosum abnormalities, while it is the opposite for KIF7. In addition, none of the KIF7 patients but all the C5orf42 patients in our cohort presented with ocular motor apraxia, which is commonly associated with the MTS in JBTS patients (Table 2 and Supplementary Table S2) [12, 27, 28]. Consistent with previous reports $[3,7,11,13,29]$, we 
observed polydactyly in half or more of the patients with KIF7 or C5orf42 pathogenic variants, which especially highlights the association of C5orf42 with polydactyly among JBTS genes. This observation also provides the clinical evidence for the possible function of C5orf42 in SHH signaling due to the well-known role of SHH pathway in limb development and association of human congenital limb defects to the defective pathway [30].

It was also remarkable that among our KIF7-positive cases, two of the Swiss patients harbored identical mutated alleles to the two previously reported Swiss patients [7]. Evaluation of their origin revealed the carriers of the splice site variant (c.2593-3 C>G) to come from the Cantons Appenzell in Northeastern Switzerland and those of the stop variant c.3001 C>T (p.(Gln1001*)) from the Canton Lucerne in central Switzerland. The estimated age of the two variants dates back to only about 2000-4000 and 1000-2000 years, respectively, corroborating their status as Swiss founder alleles. Both regions were apparently inhabited by Celtic tribes called Räter and Helvetier, respectively.

KIF7 is well known for its role in the regulation of Shh signaling in primary cilia which is crucial for limb development, tissue patterning and growth of brain structures during embryogenesis [24, 31-33]. In contrast, the protein encoded by $\mathrm{C} 5$ orf 42 has remained grossly uncharacterized. Because of its classification as a ciliopathy gene, we evaluated the primary cilia in fibroblasts of two patients with biallelic variants of C5orf42 which revealed significantly fewer and shorter cilia. We also observed significantly diminished response of these fibroblasts to the SHH agonist SAG, indicating that C5orf42 is required for efficient $\mathrm{SHH}$ signaling. In addition, silencing C5orf42 during neural development of chicken embryos revealed very similar phenotypes in commissural axon guidance to those observed after silencing Shh signaling [34, 35] which are compatible with compromised ciliary function.

Our findings of aberrant midline crossing in the CNS suggests a contribution of C5orf42-related ciliary signaling to neural circuit formation, which may explain the pathomechanism of the neurodevelopmental features seen in the patients. Although the role of ciliary signaling in neural circuit formation in the PNS has not been studied so far, our studies suggest that the mechanisms are conserved between CNS and PNS. This is in line with current knowledge in the axon guidance field that axon guidance cues and their receptors, as well as the signaling mechanisms are conserved between CNS and PNS [36]. Though, nothing is known about aberrant development of the PNS in patients, the observed changes in PNS wiring in the chicken embryo could indicate that the wiring problems also occur in the patients, where they could contribute to the observed motor problems.
Our findings are also in line with recent data from a mouse model harboring the C5orf42 S235P mutation, locating the protein to the cilia transition zone and indicating a reduced Shh response to SAG, as well as diminished number of cilia in mutated mouse embryonic fibroblasts, and absent cilia in fibroblasts of a patient with compound heterozygous C5orf42 variants [37]. Accordingly, the overlapping phenotype of C5orf42 and KIF7mutated patients may be explained by their shared role in ciliary-dependent SHH signaling. This assumption is further supported by the de novo $S H H$ variant in patient 13 with similar craniofacial features and agenesis of the corpus callosum. However, direct conclusion for the positive/ negative role of C5orf42 in regulating SHH signaling cannot be made especially based on the response of the C5orf42-mutated fibroblasts to the SAG agonist, since the repression of the pathway, though variable, would be expected to result in the narrowing of facial midline [26, 38]. This discrepancy could be due to the spatial and temporal dynamics of the pathway during development or differences in SHH responsiveness for the SAG agonist and SHH. Similar inconsistency has been reported for induction of Gli1 expression by SAG treatment in mouse embryonic fibroblasts derived from $\mathrm{Kif}^{-/-}$mice [39].

For $\mathrm{SHH}$, diverse missense and truncating variants have been reported as the most common cause of nonchromosomal HPE of different types with significant variability of penetrance and expressivity of unknown reason $[25,26]$. Therefore, although the pathogenicity of the SHH terminal frameshift variant in patient 13 may be questioned by the lack of typical HPE features, similar contrasting phenotypes have been observed for PTCHI encoding the SHH receptor. While most, probably loss-offunction variants of $\mathrm{PTCHI}$ lead to the nevoid basal cell carcinoma (Gorlin) syndrome manifesting with macrocephaly, hypertelorism and abnormal corpus callosum, some, probably activating missense variants cause HPE [40-42]. Accordingly, we suggest that some specific variants in $\mathrm{SHH}$ may mimic an ACLS phenotype.

Despite the fact that craniofacial features such as frontal prominence and broad nasal bridge have been described in a fraction of patients with JSRD [8-10], they have been less carefully documented in those with molecular diagnosis including patients with pathogenic C5orf42 variants (Table 2 and Supplementary Table S2). Our findings, in fact, highlight the overlapping craniofacial appearance of macrocephaly, prominent/broad forehead, depressed/wide nasal bridge, and hypertelorism among patients with (likely) pathogenic KIF7 and C5orf42 variants. Moreover, this clinical observation was strikingly replicated by facial midline widening after knock-down of C5orf42 in cranial NCCs of chicken embryos. Cleft lip, cleft palate, and micrognathia have been previously observed in the S235P 
mutant mice [37], corresponding to cleft lip and palate in OFDVI patients $[13,43]$. Taken together, it is likely that the aforementioned craniofacial features are due to the disturbed role of KIF7 and C5orf42 in ciliary function affecting SHH signaling, and possibly other pathways. The latter may partly explain the differences in the ciliopathy phenotypes.

In summary, our findings imply shared minimal diagnostic criteria among patients with biallelic (likely) pathogenic variants of KIF7 or C5orf42, or certain variants of $S H H$. In addition, we demonstrate the first experimental evidence for the role of C5orf42 in craniofacial development as well as axon guidance and neural circuit formation. Most likely, the effect of C5orf42 is mediated by primary cilia function, required among others in the SHH signaling pathway. Further studies will be needed to decipher the interaction partners of C5orf42 and to characterize its function in ciliary protein networks as well as ciliarydependent axon guidance.

Acknowledgements We sincerely thank the affected individuals and their families for participation and their permission to publish the results. We thank Emmanuelle Genín for providing the ESTIAGE software. This research was supported by grants from the Swiss National Science Foundation (SNF 320030_135669), Forschungskredit of the University of Zurich (grant number 54220201), and radiz-Rare Disease Initiative Zurich, clinical research priority program, University of Zurich.

\section{Compliance with ethical standards}

Conflict of interest The authors declare that they have no competing interests.

Open Access This article is licensed under a Creative Commons Attribution-NonCommercial-NoDerivatives 4.0 International License, which permits any non-commercial use, sharing, distribution and reproduction in any medium or format, as long as you give appropriate credit to the original author(s) and the source, and provide a link to the Creative Commons license. You do not have permission under this license to share adapted material derived from this article or parts of it. The images or other third party material in this article are included in the article's Creative Commons license, unless indicated otherwise in a credit line to the material. If material is not included in the article's Creative Commons license and your intended use is not permitted by statutory regulation or exceeds the permitted use, you will need to obtain permission directly from the copyright holder. To view a copy of this license, visit http://creativecommons.org/licenses/by-nc-nd/4.0/.

\section{References}

1. Schinzel A. Postaxial polydactyly, hallux duplication, absence of the corpus callosum, macrencephaly and severe mental retardation: a new syndrome? Helv Paediatr Acta. 1979;34:141-6.

2. Schinzel A, Schmid W. Hallux duplication, postaxial polydactyly, absence of the corpus callosum, severe mental retardation, and additional anomalies in two unrelated patients: a new syndrome. Am J Med Genet. 1980;6:241-9.
3. Putoux A, et al. KIF7 mutations cause fetal hydrolethalus and acrocallosal syndromes. Nat Genet. 2011;43:601-6.

4. Parisi M, Glass I. Joubert syndrome and related disorders. In: Adam MP, Ardinger HH, Pagon RA, Wallace SE, Bean LJH, Mefford HC, Stephens K, Amemiya A, Ledbetter N (eds). SourceGeneReviews ${ }^{\circledR}$ [Internet]. University of Washington: Seattle, USA, 1993-2013.

5. Valente EM, Dallapiccola B, Bertini E. Joubert syndrome and related disorders. Handb Clin Neurol. 2013;113:1879-88.

6. Courtens W, Vamos E, Christophe C, Schinzel A. Acrocallosal syndrome in an Algerian boy born to consanguineous parents: review of the literature and further delineation of the syndrome. Am J Med Genet. 1997;69:17-22.

7. Putoux A, et al. Novel KIF7 mutations extend the phenotypic spectrum of acrocallosal syndrome. J Med Genet. 2012;49:713-20.

8. Maria BL, Boltshauser E, Palmer SC, Tran TX. Clinical features and revised diagnostic criteria in Joubert syndrome. J Child Neurol. 1999;14:583-90.

9. Braddock SR, Henley KM, Maria BL. The face of Joubert syndrome: a study of dysmorphology and anthropometry. Am J Med Genet A. 2007;143A:3235-42.

10. Poretti A, et al. Delineation and diagnostic criteria of oral-facialdigital syndrome type VI. Orphanet J Rare Dis. 2012;7:4.

11. Bachmann-Gagescu R, et al. Joubert syndrome: a model for untangling recessive disorders with extreme genetic heterogeneity. J Med Genet. 2015;52:514-22.

12. Kroes HY, et al. Joubert syndrome: genotyping a Northern European patient cohort. Eur J Hum Genet. 2016;24:214-20.

13. Lopez E, et al. C5orf42 is the major gene responsible for OFD syndrome type VI. Hum Genet. 2014;133:367-77.

14. Schinzel A. The acrocallosal syndrome in first cousins: widening of the spectrum of clinical features and further support for autosomal recessive inheritance. J Med Genet. 1988;25:332-6.

15. Walsh PS, Metzger DA, Higuchi R. Chelex 100 as a medium for simple extraction of DNA for PCR-based typing from forensic material. Biotechniques. 1991;10:506-13.

16. Richards S, et al. Standards and guidelines for the interpretation of sequence variants: a joint consensus recommendation of the American college of medical genetics and genomics and the association for molecular pathology. Genet Med. 2015;17:405-24.

17. Asadollahi $\mathrm{R}$, et al. The clinical significance of small copy number variants in neurodevelopmental disorders. J Med Genet. 2014;51:677-88.

18. Rauch A, et al. Range of genetic mutations associated with severe non-syndromic sporadic intellectual disability: an exome sequencing study. Lancet. 2012;380:1674-82.

19. Genin E, Tullio-Pelet A, Begeot F, Lyonnet S, Abel L. Estimating the age of rare disease mutations: the example of Triple-A syndrome. J Med Genet. 2004;41:445-9.

20. Andermatt I, Stoeckli ET. RNAi-based gene silencing in chicken brain development. Methods Mol Biol. 2014;1082:253-66.

21. Wilson NH, Stoeckli ET. In ovo electroporation of miRNA-based plasmids in the developing neural tube and assessment of phenotypes by DiI injection in open-book preparations. J Vis Exp. 2012;68:e4384.

22. Mauti O, Domanitskaya E, Andermatt I, Sadhu R, Stoeckli ET. Semaphorin6A acts as a gate keeper between the central and the peripheral nervous system. Neural Dev. 2007;2:28.

23. Hamburger V, Hamilton HL. A series of normal stages in the development of the chick embryo. J Morphol. 1951;88:49-92.

24. Dessaud E, McMahon AP, Briscoe J. Pattern formation in the vertebrate neural tube: a sonic hedgehog morphogen-regulated transcriptional network. Development. 2008;135:2489-503.

25. Dubourg C, et al. Molecular screening of SHH, ZIC2, SIX3, and TGIF genes in patients with features of holoprosencephaly 
spectrum: mutation review and genotype-phenotype correlations. Hum Mutat. 2004;24:43-51.

26. Solomon BD, et al. Genotypic and phenotypic analysis of 396 individuals with mutations in Sonic Hedgehog. J Med Genet. 2012;49:473-9.

27. Srour M, et al. Mutations in C5ORF42 cause Joubert syndrome in the French Canadian population. Am J Hum Genet. 2012;90:693-700.

28. Srour M, et al. Joubert syndrome in French Canadians and identification of mutations in CEP104. Am $\mathrm{J}$ Hum Genet. 2015;97:744-53.

29. Romani M, et al. Oral-facial-digital syndrome type VI: is C5orf42 really the major gene? Hum Genet. 2015;134:123-6.

30. Lopez-Rios J. The many lives of SHH in limb development and evolution. Semin Cell Dev Biol. 2016;49:116-24.

31. Varjosalo M, Taipale J. Hedgehog: functions and mechanisms. Genes Dev. 2008;22:2454-72.

32. Ruat M, Roudaut H, Ferent J, Traiffort E. Hedgehog trafficking, cilia and brain functions. Differentiation. 2012;83: S97-104.

33. Goetz SC, Anderson KV. The primary cilium: a signalling centre during vertebrate development. Nat Rev Genet. 2010;11:331-44.

34. Bourikas D, et al. Sonic hedgehog guides commissural axons along the longitudinal axis of the spinal cord. Nat Neurosci. 2005;8:297-304.

35. Wilson NH, Stoeckli ET. Sonic hedgehog regulates its own receptor on postcrossing commissural axons in a glypican1dependent manner. Neuron. 2013;79:478-91.
36. Stoeckli E. Where does axon guidance lead us? F1000Research. 2017;6:78.

37. Damerla RR, et al. Novel Jbts17 mutant mouse model of Joubert syndrome with cilia transition zone defects and cerebellar and other ciliopathy related anomalies. Hum Mol Genet. 2015;24:3994-4005.

38. Bear KA, et al. Pathogenic mutations in GLI2 cause a specific phenotype that is distinct from holoprosencephaly. J Med Genet. 2014;51:413-8.

39. Liu YC, et al. The PPFIA1-PP2A protein complex promotes trafficking of Kif7 to the ciliary tip and Hedgehog signaling. Sci Signal. 2014;7:ra117.

40. Wicking $\mathrm{C}$, et al. Most germ-line mutations in the nevoid basal cell carcinoma syndrome lead to a premature termination of the PATCHED protein, and no genotype-phenotype correlations are evident. Am J Hum Genet. 1997;60:21-6.

41. Ming JE, et al. Mutations in PATCHED-1, the receptor for SONIC HEDGEHOG, are associated with holoprosencephaly. Hum Genet. 2002;110:297-301.

42. Ribeiro LA, Murray JC, Richieri-Costa A. PTCH mutations in four Brazilian patients with holoprosencephaly and in one with holoprosencephaly-like features and normal MRI. Am J Med Genet A. 2006;140:2584-6.

43. Wentzensen IM, et al. Exome sequencing identifies novel mutations in C5orf42 in patients with Joubert syndrome with oralfacial-digital anomalies. Hum Genome Var. 2015;2:15045.

\section{Affiliations}

Reza Asadollahi $^{1} \cdot$ Justin E Strauss $^{2} \cdot$ Martin Zenker $^{3} \cdot$ Oliver Beuing $^{4}{ }^{4} \cdot$ Simon Edvardson $^{5} \cdot$ Orly Elpeleg $^{6} \cdot$ Tim M Strom $^{7} \cdot$ Pascal Joset $^{1} \cdot$ Dunja Niedrist $^{1} \cdot$ Christine Otte $^{1} \cdot$ Beatrice Oneda $^{1} \cdot$ Paranchai Boonsawat $^{1} \cdot$ Silvia Azzarello-Burri $^{1} \cdot$ Deborah Bartholdi $^{1}$. Michael Papik $^{1} \cdot$ Markus Zweier $^{1} \cdot$ Cordula Haas $^{8} \cdot$ Arif B Ekici $^{9} \cdot$ Alessandra Baumer $^{1} \cdot$ Eugen Boltshauser $^{10} \cdot$ Katharina Steindl $^{1}$. Michael Nothnagel $\mathbb{D}^{11} \cdot$ Albert Schinzel $^{1} \cdot$ Esther T Stoeckli ${ }^{2,12} \cdot$ Anita Rauch $\mathbb{D}^{1,12,13}$

1 Institute of Medical Genetics, University of Zurich, SchlierenZurich, Switzerland

2 Institute of Molecular Life Sciences, University of Zurich, Zurich, Switzerland

3 Institute of Human Genetics, University Hospital Magdeburg, Magdeburg, Germany

4 Institute of Neuroradiology, Otto-von-Guericke-University Magdeburg, Magdeburg, Germany

5 Pediatric Neurology Unit, Hadassah University Hospital, Mount Scopus, Jerusalem, Israel

6 Monique and Jacques Roboh Department of Genetic Research, Hadassah-Hebrew University Medical Center, Jerusalem, Israel

7 Institute of Human Genetics, Technische Universität München,
Munich, Germany

8 Zurich Institute of Forensic Medicine, University of Zurich, Zurich, Switzerland

9 Institute of Human Genetics, Friedrich-Alexander-Universität Erlangen-Nürnberg, Erlangen, Germany

10 Department of Pediatric Neurology, University Children's Hospital, Zurich, Switzerland

11 Cologne Center for Genomics, Department of Statistical Genetics and Bioinformatics, University of Cologne, Cologne, Germany

12 Neuroscience Center Zurich, University of Zurich, Zurich, Switzerland

13 Zurich Center of Integrative Human Physiology, University of Zurich, Zurich, Switzerland 